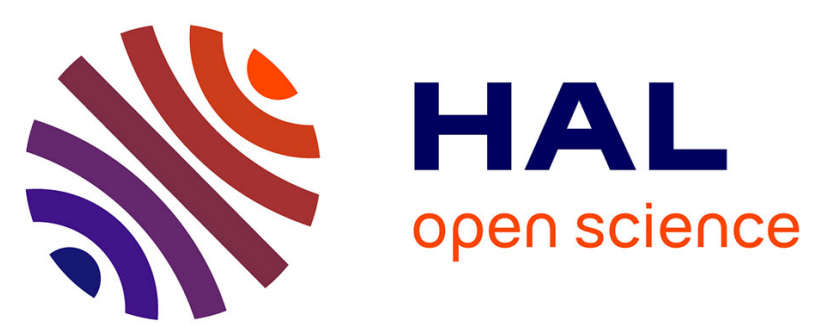

\title{
A mode matching approach for modeling two dimensional porous grating with infinitely rigid or soft inclusions
}

Benoit Nennig, Y. Renou, J.-P. Groby, Yves Aurégan

\section{- To cite this version:}

Benoit Nennig, Y. Renou, J.-P. Groby, Yves Aurégan. A mode matching approach for modeling two dimensional porous grating with infinitely rigid or soft inclusions. Journal of the Acoustical Society of America, 2012, 131 (5), pp.3841-3852. 10.1121/1.3693655 . hal-01179031

\section{HAL Id: hal-01179031 \\ https://hal.science/hal-01179031}

Submitted on 22 Jul 2015

HAL is a multi-disciplinary open access archive for the deposit and dissemination of scientific research documents, whether they are published or not. The documents may come from teaching and research institutions in France or abroad, or from public or private research centers.
L'archive ouverte pluridisciplinaire HAL, est destinée au dépôt et à la diffusion de documents scientifiques de niveau recherche, publiés ou non, émanant des établissements d'enseignement et de recherche français ou étrangers, des laboratoires publics ou privés. 


\title{
A mode matching approach for modeling two dimensional porous grating with infinitely rigid or soft inclusions
}

\author{
Benoit Nennig, a) Ygaäl Renou, Jean-Philippe Groby, and Yves Aurégan \\ Laboratoire d'Acoustique de l'Université du Maine, UMR6613 CNRS/Univ. du Maine, \\ Avenue Olivier Messiaen, 72085 Le Mans Cedex 9, France
}

(Dated: February 3, 2012, Version 5.1)

\begin{abstract}
This work investigates the acoustical properties of a multilayer porous material in which periodic inclusions are embedded. The material is assumed to be backed by a rigid wall. Most of the studies performed in this field used the multipole method and are limited to circular shape inclusions. Here, a mode matching approach, more convenient for a layered system, is adopted. The inclusions can be in the form of rigid scatterers of an arbitrary shape, in the form of an air-filled cavity or in the form of a porous medium with contrasting properties. The computational approach is validated on simple geometries against other numerical schemes and with experimental results obtained in an anechoic room on a rigid grating embedded in a porous material made of $2 \mathrm{~mm}$ glass beads. The method is used to study the acoustic absorption behavior of this class of materials in the low frequency range and at a range of angles of incidence.
\end{abstract}

PACS numbers: 43.55.Ev, 43.20.Fn, 43.20.Ks, 43.20.Gp

\section{INTRODUCTION}

Porous materials are widely used in noise control applications because of their good sound absorbing properties in the middle and high frequency range. However, their low frequency sound absorbing properties are limited when the layer thickness becomes considerably less than the wavelength. A common practical solution here has been to make use of multilayer materials ${ }^{1}$ to reduce the thickness of the overall porous structure and to improve its acoustic absorption properties. Another alternative solution is to embed inclusions into the porous layer to provide additional energy scattering and dissipation mechanisms ${ }^{10}$. The purpose of this paper is to investigate more systematically the sound absorption properties of embedded periodic inclusions in a porous layer with an numerical technique. The proposed approach is based on the mode matching method.

The idea of using inclusions in porous media is not entirely new. Perforations (i.e. air inclusion) have been exploited in the past to improve the sound absorption of porous material with high flow resistivity. Two distinct approaches where used in normal incidence for the so called double porosity material : (i) using finite element method (FEM $)^{2-4}$ possibly combined with a topological optimization algorithm $^{5}$; (ii) using homogenization ${ }^{6,7}$. This model has recently been adapted by Gourdon et al. ${ }^{8}$ to account for porous material inclusions instead of air cavities. Some noticeable enhancements of the vibroacoustical properties of the porous materials have been obtained for transparency applications.

All previous models are limited to normal incidence

a)Electronic address: benoit.nennig@supmeca.fr; Present address: Laboratoire d'Ingénierie des Systèmes Mécaniques et des Matériaux (LISMMA), SUPMECA, 3 rue Fernand Hainaut, 93407 SaintOuen, France. but the oblique case may be investigated thanks to the Floquet-Bloch formalism assuming periodic heterogeneities. This idea was investigated by Groby et al. in Ref. 10 for the prediction of the acoustic absorption properties of an heterogeneous porous layer with hard backing. The latter work is mainly concerned with circular rigid inclusions.

In these and other works only few attempts have been made to investigate the effects of an rigid inclusion shape on the acoustic absorption coefficient. In this regard, Allard et al. ${ }^{12}$ have proposed a layered mode matching approach to study arbitrary corrugated porous materials. Nevertheless, this method have been developed to predict the effect of porous inclusions. When dealing with rigid inclusions, the model must be improved in order to take into account more accurately the presence of geometrical singularity such as corners.

One possibility is to make use of orthogonal polynomial basis and in particular their relation to singular weighted function $^{14}$ (Chap. 22) and then to combine them with the modal expansion that will take into account the velocity singularity at corners. A more general method ${ }^{15}$ exists which is directly relevant to the solution of the above problem. The idea behind this method has been exploited by Evans and Fernyhough ${ }^{16}$ for waves along a periodic coastline or by Homentcovschi et al. ${ }^{17}$ for waveguide discontinuity problems. This approach is extensively used throughout this paper.

The focus of this paper is on the analysis of heterogeneous inclusions of arbitrary shape embedded in homogeneous porous layers. These heterogeneities can either be air cavities, contrasting porous fillings or rigid scatterers. The proposed layered mode matching scheme is somewhat similar to that presented in Ref. 12 but is able to model properly rigid inclusions.

The method can be used to tackle a generalized case of multilayer structures were all layers can be treated as heterogeneous. This approach seems to be a good com- 
promise between the computational time and configuration diversity when benchmarked against the finite element method. Compared to homogenization, there is no constraint for the ratio of the size of the heterogeneities to the acoustic wavelength which the method is able to consider.

The present paper is organized as follows. The proposed enhanced mode matching method is presented in Section II. In section III, the validity and the accuracy of this method is studied with comparison against other computation mathods and measurements. The experimental data are obtained for a periodic gratings with square rigid inclusion embedded in a porous material composed of loose glass beads. In the last section, various numerical examples of increasing complexity are presented.

\section{FORMULATION OF THE METHOD}

\section{A. Problem statement}

We consider that an acoustic plane wave is incident at the oblique angle of incidence on a system of porous layers. We assume that system of layers is periodic in the direction $x_{1}$ and that the spatial period is $d$ (see Fig. 1). We assume that the total number of layers stacked up in the direction $x_{2}$ is $N_{\mathscr{L}}$. We allow for some heterogeneities to be present within this stack so that a horizontal layer can be split into several sub-layers to represent these heterogeneities as illustrated in Fig. 1. Specifically, we assume that when the propagating wave encounters a pair of vertical rigid walls in the layer $\mathscr{L}^{i}$, then the layer must be split, into $N_{\mathscr{C}}^{i}$ cells such $\mathscr{L}^{i}=\bigcup_{j=1}^{N_{\mathscr{C}}^{i}} \mathscr{C}^{i, j}$. We also allow for different materials $\Omega^{i, j}$ to be included in each cell. A new cell $\mathscr{C}^{i, j}$ have to be added each time independent wave propagation can occurred in a layer $\mathscr{L}^{i}$, i.e. when vertical rigid walls are present. The interface $\Gamma^{i}\left(i=0, \ldots, N_{\mathscr{L}}\right)$ between two layers can be inhomogeneous. It can also be partly rigid interface. The surrounding domain $\Omega^{0}$ is filled with air with the sound speed $c^{0}$ and density $\rho^{0}$.

The skeleton of the porous material is considered to be infinitely rigid, therefore the Champoux-Allard-Johnson equivalent fluid model ${ }^{1}$ can be used to predict the equivalent bulk modulus $K^{i, j}$ and the dynamic density $\rho^{i, j}$ (see Appendix A for details). In this case, the wave speed in the domain $\Omega^{i, j}$ is given by the ratio $c^{i, j}=\sqrt{K^{i, j} / \rho^{i, j}}$. The expression for the acoustic pressure, $p$, in the equivalent fluid (resp. in the fluid) in each of the system domains, $\Omega^{i, j}$, satisfies the harmonic wave equation (the time dependence $\mathrm{e}^{-\mathrm{i} \omega t}$ is assumed here)

$$
\Delta p(\mathbf{x})+\left(k^{i, j}\right)^{2} p(\mathbf{x})=0,
$$

where the wavenumber is defined by $k^{i, j}=\omega / c^{i, j}$.

Since the geometry is periodic along $x_{1}$ and the excitation is by a plane wave, $p^{\text {inc }}=A_{0} \mathrm{e}^{\mathrm{ik} \cdot \mathbf{x}}$, each physical variable within this system $X$ should satisfy the FloquetBloch relation

$$
X\left(x_{1}+d, x_{2}\right)=X\left(x_{1}, x_{2}\right) \mathrm{e}^{\mathrm{i} k_{1} d},
$$

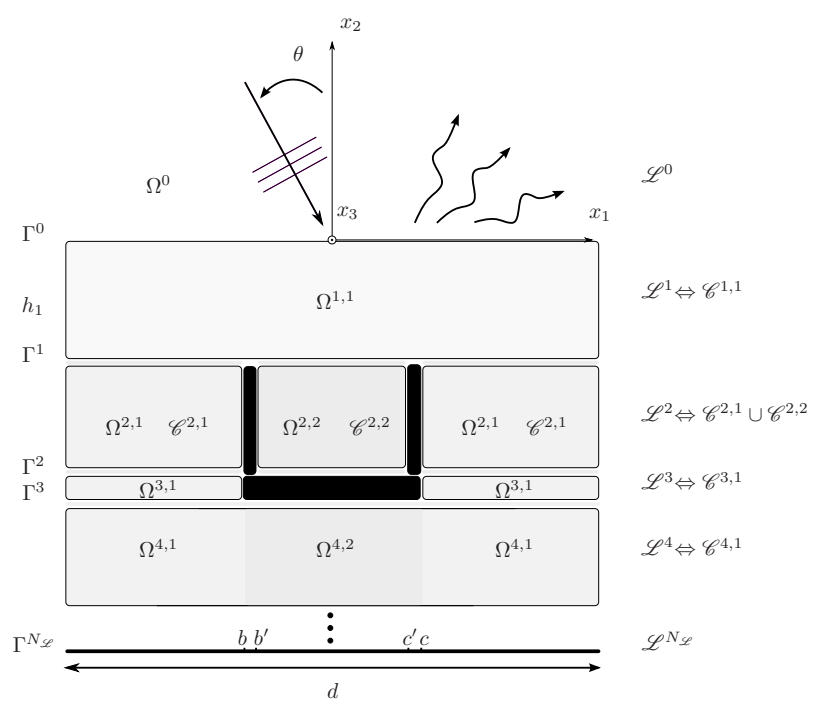

FIG. 1. Geometry of the periodic cell.

where the incident wavenumber is $\mathbf{k}=\left(k_{1}, k_{2}\right)=k^{0}$. $(\sin \theta,-\cos \theta)$. For the sake of conciseness, $\vartheta=\mathrm{e}^{\mathrm{i} k_{1} d}$ and $\vartheta^{*}=\mathrm{e}^{-\mathrm{i} k_{1} d}$ will be used.

We are looking to predict the sound pressure field anywhere in the system of porous layers shown in Fig. 1. As the problem is separable in each layer $\mathscr{L}^{i}$, the pressure can be written in the form

$$
p^{i}(\mathbf{x})=\chi\left(x_{1}\right) \mathcal{Y}\left(x_{2}\right), \forall \mathbf{x} \in \mathscr{L}^{i} .
$$

In the surrounding fluid domain $\Omega^{0}$, the radiation condition, Floquet condition (2) and the separation of variables (3) lead to ${ }^{18}$

$$
p^{0}(\mathbf{x})=\sum_{n \in \mathbb{Z}} A_{n}^{0} \frac{1}{\sqrt{d}} \mathrm{e}^{\mathrm{i} \alpha_{n}^{0} x_{1}} \mathrm{e}^{\mathrm{i} \beta_{n}^{0} x_{2}}, \forall \mathbf{x} \in \Omega^{0},
$$

where $\alpha_{n}^{0}=k_{1}+n \frac{2 \pi}{d}$ and $\beta_{n}^{0}=\sqrt{\left(k^{0}\right)^{2}-\left(\alpha_{n}^{0}\right)^{2}}$. The axial wavenumber $\beta_{n}^{0}$ can be real (i.e. a propagating wave) or imaginary (i.e. an evanescent wave). When an incident wave impinges on a periodic, non-planar surface, the scattered waves are spread in discrete directions which depends whether a mode is propagating or not. If $\beta_{n}^{0}=0$, the mode is said to be resonant ${ }^{18}$, and scattered waves can propagate along the material interface.

In the case of a heterogeneous absorbing material, the above formalism needs a more detailed explanation. The sound pressure in a porous layer $\mathscr{L}^{i}$ which consists of several sub-domains is effectively a combination of the sound pressures in all the cells $\mathscr{C}^{i, j}$ of this layer, i.e.

$$
p^{i}(\mathbf{x})=\bigcup_{j=1}^{N_{\mathscr{C}}^{i}} p^{i, j}(\mathbf{x}), \forall \mathbf{x} \in \mathscr{L}^{i}
$$

where, the sound pressure field in the cell $\mathscr{C}^{i, j}$ is sought using the modal expansion

$$
\begin{aligned}
p^{i, j}(\mathbf{x})=\sum_{n \in \mathbb{N}} & \left(A_{n}^{i, j} \chi_{n}^{i, j}\left(x_{1}\right) \mathrm{e}^{\mathrm{i} \beta_{n}^{i, j} x_{2}}\right. \\
& \left.+B_{n}^{i, j} \chi_{n}^{i, j}\left(x_{1}\right) \mathrm{e}^{-\mathrm{i} \beta_{n}^{i, j} x_{2}}\right), \forall \mathbf{x} \in \mathscr{C}^{i, j} .
\end{aligned}
$$


Here, $A_{n}^{i, j}$ and $B_{n}^{i, j}$ are the $x_{2}>0$ and $x_{2}<0$ traveling wave amplitudes, respectively. $\chi_{n}^{i, j}$ and $\beta_{n}^{i, j}$ are the eigenfunctions and the eigenvalues, respectively. The latter satisfy the Helmholtz equation (1) and the FloquetBloch relation (2). Details on their calculation are given in the following section.

The velocity along $x_{2}$ axis, $v^{i, j}=\frac{1}{i \omega \rho^{i, j}} \partial_{x_{2}} p^{i, j}$, is given by the following series

$$
\begin{aligned}
v^{i, j}(\mathbf{x})=\sum_{n \in \mathbb{N}} & \left(A_{n}^{i, j} \chi_{n, v}^{i, j}\left(x_{1}\right) \mathrm{e}^{\mathrm{i} \beta_{n}^{i, j} x_{2}}\right. \\
& \left.-B_{n}^{i, j} \chi_{n, v}^{i, j}\left(x_{1}\right) \mathrm{e}^{-\mathrm{i} \beta_{n}^{i, j} x_{2}}\right), \forall \mathbf{x} \in \mathscr{C}^{i, j},
\end{aligned}
$$

where $\chi_{n, v}^{i, j}\left(x_{1}\right)=\frac{1}{\omega \rho^{i, j}} \beta^{i, j} \chi_{n}^{i, j}\left(x_{1}\right)$. Similarly to the sound pressure, the velocity in the layer $\mathscr{L}^{i}$ is defined by

$$
v^{i}(\mathbf{x})=\bigcup_{j=1}^{N_{\mathscr{C}}^{i}} v^{i, j}(\mathbf{x}), \forall \mathbf{x} \in \mathscr{L}^{i}
$$

In the above summations, the origin of the $x_{2}$ is fixed at the upper material surface $\Gamma^{0}$.

\section{B. Sound field in a periodic cell}

Here we focus on a periodic cell which is illustrated in Fig. 1. We will determine the eigenmodes and use them to predict the sound pressure and velocity with the normal mode decomposition method proposed in the previous section.

\section{Simple layer}

Let us consider the first and unique homogeneous cell within the layer $\mathscr{L}^{1}$ (see Fig. 1). As previously mentioned, all the physical variables must satisfy the 1-D Helmholtz equation (1). This yields

$$
\chi\left(x_{1}\right)=A \mathrm{e}^{\mathrm{i} \alpha x_{1}}+B \mathrm{e}^{-\mathrm{i} \alpha x_{1}} .
$$

Applying the Floquet-Bloch relation (2) on the pressure and the velocity ${ }^{19}$ (see section 3.2.2), we get

$$
\begin{aligned}
\chi\left(x_{1}+d\right) & =\chi\left(x_{1}\right) \vartheta, \\
\partial_{x_{1}} \chi\left(x_{1}+d\right) & =\partial_{x_{1}} \chi\left(x_{1}\right) \vartheta,
\end{aligned}
$$

leading to the eigenvalue problem

$$
\left(\begin{array}{cc}
\vartheta-\mathrm{e}^{\mathrm{i} \alpha d} & \vartheta-\mathrm{e}^{-\mathrm{i} \alpha d} \\
\vartheta-\mathrm{e}^{\mathrm{i} \alpha d} & -\vartheta+\mathrm{e}^{-\mathrm{i} \alpha d}
\end{array}\right)\left(\begin{array}{l}
A \\
B
\end{array}\right)=\left(\begin{array}{l}
0 \\
0
\end{array}\right) .
$$

The dispersion equation is

$$
\left(1-\vartheta^{2}\right)+2 \vartheta \cos \alpha d=0
$$

and its solutions are $\alpha_{n}= \pm\left(n \frac{2 \pi}{d}+k_{1}\right)$ with $n \in \mathbb{Z}$. The wavenumber along $x_{2}$ is given by $\beta_{n}=\sqrt{\left(k^{i, j}\right)^{2}-\alpha_{n}^{2}}$. Therefore, only the positive $\alpha_{n}$ is needed to determine the corresponding wavenumbers $\beta_{n}$. The eigenfunctions are given by

$$
\chi_{n}\left(x_{1}\right)=\frac{1}{\sqrt{d}} \mathrm{e}^{\mathrm{i}\left( \pm k_{1}+n \frac{2 \pi}{d}\right) x_{1}} .
$$

In this special case $\chi_{n}$ represent propagating waves as in $\Omega^{0}$ along $x_{1}$.

\section{Sound propagation in a layer with a rigid inclusion}

Consider now the cell $\mathscr{C}^{2,1}$ which belongs to the layer $\mathscr{L}^{2}$ (see Fig. 1). This domain is split into two parts because of the presence of a rigid inclusion at $x_{1} \in[b, c]$. Two boundary conditions which need to be added to Eq.(10) are

$$
\begin{aligned}
& \partial_{x_{1}} \chi(b)=0 \\
& \partial_{x_{1}} \chi(c)=0 .
\end{aligned}
$$

It is necessary for the eigenmodes to satisfy the 1-D Helmholtz equation (1), so that

$$
\chi\left(x_{1}\right)= \begin{cases}A \mathrm{e}^{\mathrm{i} \alpha x_{1}}+B \mathrm{e}^{-\mathrm{i} \alpha x_{1}}, & \text { if } x_{1} \in[0, b], \\ A^{\prime} \mathrm{e}^{\mathrm{i} \alpha x_{1}}+B^{\prime} \mathrm{e}^{-\mathrm{i} \alpha x_{1}}, & \text { if } x_{1} \in[c, d] .\end{cases}
$$

Applying the boundary conditions from Eq. (14) and Eq. (10) leads to $B=A \mathrm{e}^{2 \mathrm{i} \alpha b}, B^{\prime}=A^{\prime} \mathrm{e}^{2 \mathrm{i} \alpha c}$ and

$$
\left(\begin{array}{c}
\left(1+\mathrm{e}^{2 \mathrm{i} \alpha b}\right) \vartheta-\left(\vartheta+\mathrm{e}^{2 \mathrm{i} \alpha c}\right) \\
\left(1-\mathrm{e}^{2 \mathrm{i} \alpha b}\right) \vartheta-\left(\vartheta-\mathrm{e}^{2 \mathrm{i} \alpha c}\right) \\
\vartheta
\end{array}\right)\left(\begin{array}{c}
A \\
A^{\prime}
\end{array}\right)=\left(\begin{array}{l}
0 \\
0
\end{array}\right) .
$$

The determinant of this system yields $\sin \alpha(d-a)=0$ with $a=c-b$. The solutions are $\alpha_{n}=\frac{n \pi}{(d-a)}$ as in the normal incidence case. However, the eigenfunctions are modified by the Floquet-Bloch relation and we get $A^{\prime}=(-1)^{n} \vartheta \mathrm{e}^{-\mathrm{i} \alpha_{1} a} A$. Now let us focus on the cell $\mathscr{C}^{2,2}$ embedded in the layer $\mathscr{L}^{2}$. The solution here is somewhat simpler because the eigenproblem is independent of the periodic conditions. In this case, the eigenmodes are similar to those determined for the case of a standard rigid cavity ${ }^{20}$, i.e. $\chi_{n}\left(x_{1}\right)=\cos \alpha_{n} x_{1}$ for $x_{1} \in\left[b^{\prime}, c^{\prime}\right]$ with $\alpha_{n}=n \pi / a^{\prime}$ letting $a^{\prime}=c^{\prime}-b^{\prime}$.

\section{Sound propagation in a layer with a fluid/porous inclusion}

Consider now the cell $\mathscr{C}^{4,1}$ which belongs to the layer $\mathscr{L}^{4}$ (see Fig. 1). This cell is split into two parts because of the presence of another material in $\Omega^{4,2}$, which can be a fluid or a porous material with contrasting properties. The boundary conditions are given by the Floquet-Bloch relation (10) and the continuity conditions at a fluid/fluid interface are

$$
\begin{array}{ll}
p^{4,1}(b)=p^{4,2}(b), & p^{4,1}(c)=p^{4,2}(c), \\
u^{4,1}(b)=u^{4,2}(b), & u^{4,1}(c)=u^{4,2}(c) .
\end{array}
$$

Here $u^{i, j}=\frac{1}{\mathrm{i} \omega \rho^{i, j}} \partial_{x_{1}} p^{i, j}$ is the acoustic velocity along the $x_{1}$ axis. The corresponding eigenmodes are determined 
from

$$
\chi\left(x_{1}\right)= \begin{cases}A_{1} \mathrm{e}^{\mathrm{i} \alpha_{1} x_{1}}+B_{1} \mathrm{e}^{-\mathrm{i} \alpha_{1} x_{1}} & \text { If } x_{1} \in[0, b[, \\ A_{2} \mathrm{e}^{\mathrm{i} \alpha_{2} x_{1}}+B_{2} \mathrm{e}^{-\mathrm{i} \alpha_{2} x_{1}} & \text { If } x_{1} \in[b, c[, \\ A_{1}^{\prime} \mathrm{e}^{\mathrm{i} \alpha_{1} x_{1}}+B_{1}^{\prime} \mathrm{e}^{-\mathrm{i} \alpha_{1} x_{1}} & \text { If } x_{1} \in[c, d] .\end{cases}
$$

From (17) $A^{\prime}=A \mathrm{e}^{\mathrm{i}\left(k_{1}-\alpha_{1}\right) d}$, and $B^{\prime}=B \mathrm{e}^{\mathrm{i}\left(k_{1}+\alpha_{1}\right) d}$ it follows that

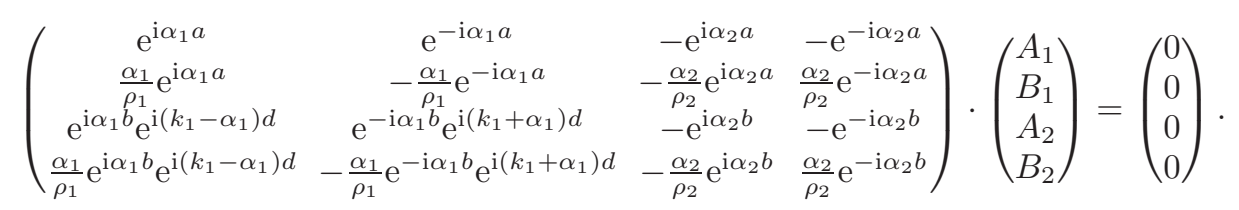

After the substitution $\alpha_{1}=\sqrt{\left(k^{4,1}\right)^{2}-\left(\beta^{4}\right)^{2}}$ and $\alpha_{2}=$ $\sqrt{\left(k^{4,2}\right)^{2}-\left(\beta^{4}\right)^{2}}$, the determinant of this system yields the dispersion equation with respect to $\beta^{4}$. In this case, the solutions are not known and numerical methods must be used. We use the algorithm proposed in Ref. 21, which requires an analytic dispersion equation but no initial guess thanks to the winding number (WN) technique. This technique is used to find the roots $\beta_{n}^{i}$ at the first frequency, and these roots are then used as an initial guess for the next frequency step using the Newton-Raphson (NR) algorithm, which is faster but less robust. If the NR algorithm fails, the WN technique is then used instead. This strategy takes the advantage of the both algorithms in terms of its robustness and computational time. It is worth noting that the derivatives are computed analytically to fully take advantage of the NR quadratic convergence property. Once the eigenvalues are found, the eigenmodes can be obtained with a singular value decomposition or another technique. The eigenfunctions are orthogonal if the cell is symmetric ${ }^{12}$. This property is not used directly in this paper but contributes to the robustness of the proposed method. Further details on the orthogonality problem arising in dissipative media are given in Refs. 22, 23.

\section{Mode matching strategy}

At each layer interface $\Gamma^{i}\left(i=0, \ldots, N_{\mathscr{L}}-1\right)$, the condition of the continuity of the pressure and of the normal velocity apply. On the rigid backing, i.e. at interface $\Gamma^{N_{\mathscr{L}}}$, the normal velocity must vanish. The modal expansions, given in Eqs. (5) and (8) can be truncated to $N$ first terms for all the layers. The correspondence $n \leftrightarrow \beta_{n}^{i, j}$ is given by organizing the imaginary part of the eigenvalues in ascending order so that the lowest attenuation modes are always included in the series. In this work two mode matching schemes have been used: (i) the classical mode matching scheme ${ }^{12}$ was used in the case of soft inclusions such as air or another porous material; (ii) the mode re-expansion method ${ }^{16,17}$ was used in the case of rigid inclusions. These two numerical schemes are detailed below.

\section{The classical mode matching scheme}

Let us defined

$$
\chi^{i}(\mathbf{x})=\bigcup_{j=1}^{N_{\bullet}^{i}} \chi_{n}^{i, j(\mathbf{x})}, \forall \mathbf{x} \in \mathscr{L}^{i},
$$

to be the expression for the $n$-th modal profile along the material interface. Matching conditions between the layers are imposed in a weighted sense, i.e. we proceed by choosing a weighting function and then integrate over the interface $\Gamma^{i}$

$$
\begin{gathered}
\int_{0}^{d} \chi_{m}^{i \dagger}\left(p^{i}-p^{i+1}\right) \mathrm{d} x_{1}=0 \\
\int_{0}^{d} \chi_{m, v}^{i+1}{ }^{\dagger}\left(v^{i}-v^{i+1}\right) \mathrm{d} x_{1}=0 .
\end{gathered}
$$

Here, the weighting functions $\chi_{m}^{i}$ and $\chi_{m, v}^{i}+1$ are the complex conjugate (denoted by ${ }^{\dagger}$ ) eigenfunction of the layer $i$ and of the layer $i+1$, respectively ${ }^{26}$.

Using (5) to (8) leads to the overlap integrals matrices

$$
\left(\mathbf{P}^{(j)}\right)_{m, n}=\int_{0}^{d}\left(\chi_{m}^{i}\right)^{\dagger} \chi_{n}^{j} \mathrm{~d} x_{1}
$$

and

$$
\left(\mathbf{V}^{(j)}\right)_{m, n}=\int_{0}^{d}\left(\chi_{m, v}^{i+1}\right)^{\dagger} \chi_{n, v}^{j} \mathrm{~d} x_{1}
$$

where the subscripts $m$ and $n$ are in the $[0, N]$ range. For generality, these integrals are computed numerically using the Gauss-Legendre quadrature rule, although their analytical forms for some special cases can be found in Ref. 12. We note that $8 N$ Gaussian quadrature points are used here.

Using previous equations, the scattering system at the interface $\Gamma_{i}$ becomes

$$
\mathbf{X}^{i} \mathbf{E}_{X}\left(\begin{array}{c}
\mathbf{A}^{i} \\
\mathbf{B}^{i+1}
\end{array}\right)=\mathbf{Y}^{i} \mathbf{E}_{Y}\left(\begin{array}{c}
\mathbf{B}^{i} \\
\mathbf{A}^{i+1}
\end{array}\right)
$$

where the vectors $\mathbf{A}^{i}, \mathbf{B}^{i}$ contain the modal amplitudes and the diagonal matrices $\mathbf{E}_{X}$ and $\mathbf{E}_{Y}$ contain the phase 


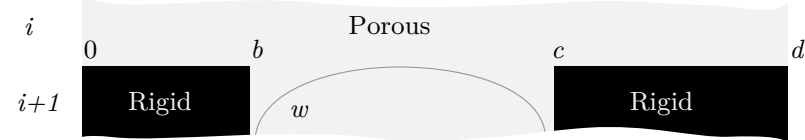

(a)

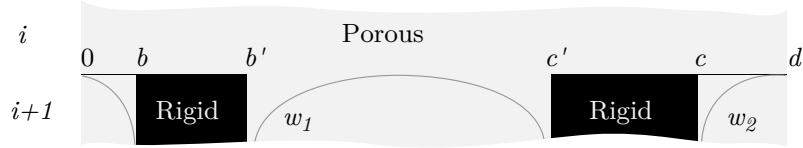

(b)

FIG. 2. Details of two rigid-soft interfaces. With one periodic inclusion (a), with two periodic inclusions (b).

factor, i.e.

$$
\begin{aligned}
& \mathbf{E}_{X}=\operatorname{diag}\left(\mathrm{e}^{\mathrm{i} \beta_{1}^{i} h_{i}} \ldots \mathrm{e}^{\mathrm{i} \beta_{N}^{i} h_{i}}, \mathrm{e}^{-\mathrm{i} \beta_{1}^{i+1} h_{i}} \ldots \mathrm{e}^{-\mathrm{i} \beta_{N}^{i+1} h_{i}}\right), \\
& \mathbf{E}_{Y}=\operatorname{diag}\left(\mathrm{e}^{-\mathrm{i} \beta_{1}^{i} h_{i}} \ldots \mathrm{e}^{-\mathrm{i} \beta_{N}^{i} h_{i}}, \mathrm{e}^{\mathrm{i} \beta_{1}^{i+1} h_{i}} \ldots \mathrm{e}^{\mathrm{i} \beta_{N}^{i+1} h_{i}}\right) .
\end{aligned}
$$

The matrices $\mathbf{X}^{i}$ and $\mathbf{Y}^{i}$ in the above equations are given by

$$
\begin{aligned}
& \mathbf{X}^{i}=\left(\begin{array}{cc}
\mathbf{P}^{(i)} & -\mathbf{P}^{(i+1)} \\
\mathbf{V}^{(i)} & \mathbf{V}^{(i+1)}
\end{array}\right), \\
& \mathbf{Y}^{i}=\left(\begin{array}{cc}
-\mathbf{P}^{(i)} & \mathbf{P}^{(i+1)} \\
\mathbf{V}^{(i)} & \mathbf{V}^{(i+1)}
\end{array}\right),
\end{aligned}
$$

respectively.

\section{The re-expansion matching scheme}

When rigid inclusions are present, the re-expansion matching scheme is more robust than the classical one. We follow the approach used by Homentcovschi ${ }^{17}$ for the computation of the scattering matrix for the waveguide discontinuity problem. This method is detailed for the case with one inclusion per layer as illustrated on Fig. 2. If more rigid inclusions are present, then the method can be easily extended.

The first step is to introduce an auxiliary normal velocity field at the interface $\Gamma^{i}$ for $x_{1} \in[b, c]$ under the series expansion

$$
\tilde{v}\left(x_{1}\right)=w\left(x_{1}\right) \sum_{k=0}^{K} V_{k} C_{k}^{\nu}\left(x_{1}\right),
$$

with the weight function

$$
w\left(x_{1}\right)=\left[\left(c-x_{1}\right)\left(x_{1}-b\right)\right]^{\nu-1 / 2} .
$$

The most important thing is the singular behavior of $w\left(x_{1}\right)$ for $x_{1}=b$ and $x_{1}=c$, which can be tuned with $\nu$ to get the singularity order of the velocity near a corner ${ }^{17}$. We suppose that this singular behavior remains the same for a rigid corner embedded in a porous media or in air, i.e. $\nu=1 / 6$. Here $V_{k}$ are some new auxiliary unknowns, $C_{k}^{\nu}$ are the Gegenbauer polynomials ${ }^{14}$ (Chap. 22) defined for $x_{1} \in[b, c]$, which are orthogonal with respect to the weight function $w\left(x_{1}\right)$.

Matching conditions between the layers are imposed in a weighted sense, i.e. we proceed by choosing a weighting function and then integrate over the interface $\Gamma^{i}$. For the pressure continuity condition, the weighting function is $w\left(x_{1}\right) C_{k}^{\nu}\left(x_{1}\right)$. This yields

$$
\int_{\gamma_{i} \cap \gamma_{i+1}} w C_{k}^{\nu} p^{i} \mathrm{~d} x_{1}=\int_{\gamma_{i} \cap \gamma_{i+1}} w C_{k}^{\nu} p^{i+1} \mathrm{~d} x_{1} .
$$

The integration support is obviously limited to $\gamma_{i} \cap \gamma_{i+1}$, where $\gamma_{i}$ stands for the support of the modal profile of the layer $\mathscr{L}^{i}$. Then, the velocity continuity is applied in two steps: imposing $\tilde{v}=v^{i}$ and $\tilde{v}=v^{i+1}$ and then by taking the projection of the velocities and their corresponding layer eigenmodes

$$
\begin{aligned}
\int_{\gamma_{i}} \chi_{m}^{i}{ }^{\dagger} v^{i} \mathrm{~d} x_{1} & =\int_{\gamma_{i} \cap \gamma_{i+1}} \chi_{m}^{i \dagger} \tilde{v}^{i} \mathrm{~d} x_{1}, \\
\int_{\gamma_{i+1}} \chi_{m}^{i+1^{\dagger}} v^{i+1} \mathrm{~d} x_{1} & =\int_{\gamma_{i} \cap \gamma_{i+1}} \chi_{m}^{i+1^{\dagger}} \tilde{v}^{i} \mathrm{~d} x_{1} .
\end{aligned}
$$

In the matrix form it reads as

$$
\begin{aligned}
\mathbf{Y}_{i}\left(\tilde{\mathbf{B}}^{i}-\tilde{\mathbf{A}}^{i}\right) & =\mathbf{D}_{i}^{\dagger} \mathbf{V} \\
\mathbf{Y}_{i+1}\left(\tilde{\mathbf{B}}^{i+1}-\tilde{\mathbf{A}}^{i+1}\right) & =\mathbf{D}_{i+1}^{\dagger} \mathbf{V} \\
\mathbf{D}_{i}\left(\tilde{\mathbf{B}}^{i}+\tilde{\mathbf{A}}^{i}\right) & =\mathbf{D}_{i+1}\left(\tilde{\mathbf{B}}^{i+1}+\tilde{\mathbf{A}}^{i+1}\right)
\end{aligned}
$$

where ${ }^{\dagger}$ stands for the conjugate transpose matrix, $\left(\tilde{\mathbf{A}}^{i}\right)_{n}=A_{n}^{i} \mathrm{e}^{\mathrm{i} \beta_{n}^{i} h_{i}}$ and $\left(\tilde{\mathbf{B}}^{i}\right)_{n}=B_{n}^{i} \mathrm{e}^{-\mathrm{i} \beta_{n}^{i} h_{i}}$ contain the modal amplitudes, $\mathbf{V}^{i}$ contain the new auxiliary unknowns and

$$
\begin{aligned}
\left(\mathbf{D}_{i}\right)_{k, n} & =\int_{\gamma_{i} \cap \gamma_{i+1}} w C_{k}^{\nu} \chi_{n}^{i} \mathrm{~d} x_{1} \\
\left(\mathbf{Y}_{i}\right)_{m, n} & =\frac{\beta_{n}^{i}}{\omega \rho^{i}} \int_{\gamma_{i}} \chi_{m}^{i \dagger} \chi_{n}^{i} \mathrm{~d} x_{1} .
\end{aligned}
$$

The above integrals are computed numerically. The choice of the numerical quadrature adopted here is essentially motivated by its reletive simplicity and numerical stability. The indices $m, n$ and $k$ belong to $[0, N]$.

The use of the numerical Gauss-Gegenbauer quadrature rule ${ }^{24}$ which accounts for the weight function $w$ singularity is very efficient when $8 N$ Gauss quadrature points are used. In this procedure the recursive definition of the Gegenbauer polynomials ${ }^{14}$ (Table 22.7) can be used to limit the round-off error.

The expressions for the scattering matrices

$$
\mathbf{E}_{X}\left(\begin{array}{c}
\mathbf{A}^{i} \\
\mathbf{B}^{i+1}
\end{array}\right)=\left(\begin{array}{cc}
\mathbf{S}_{i}^{11} & \mathbf{S}_{i}^{12} \\
\mathbf{S}_{i}^{21} & \mathbf{S}_{i}^{22}
\end{array}\right) \mathbf{E}_{Y}\left(\begin{array}{c}
\mathbf{B}^{i} \\
\mathbf{A}^{i+1}
\end{array}\right)
$$


relating the incident and reflected waves at each interface $\Gamma_{i}$ have been given in Ref. 17 and are recalled here as

$$
\begin{aligned}
& \mathbf{S}_{i}^{11}=\left(\mathbf{H}_{i}+\mathbf{D}_{i}\right)^{-1}\left(\mathbf{H}_{i}-\mathbf{D}_{i}\right), \\
& \mathbf{S}_{i}^{22}=\left(\mathbf{H}_{i+1}+\mathbf{D}_{i+1}\right)^{-1}\left(\mathbf{H}_{i+1}-\mathbf{D}_{i+1}\right), \\
& \mathbf{S}_{i}^{21}=2\left(\mathbf{D}_{i+1}\right)^{-1} \mathbf{D}_{i}\left(\mathbf{H}_{i}+\mathbf{D}_{i}\right)^{-1} \mathbf{H}_{i}, \\
& \mathbf{S}_{i}^{12}=2\left(\mathbf{D}_{i}\right)^{-1} \mathbf{D}_{i+1}\left(\mathbf{H}_{i+1}+\mathbf{D}_{i+1}\right)^{-1} \mathbf{H}_{i+1},
\end{aligned}
$$

with

$$
\begin{aligned}
\mathbf{H}_{i} & =\mathbf{D}_{i+1} \mathbf{Y}_{i+1}^{-1} \mathbf{D}_{i+1}^{\dagger}\left(\mathbf{D}_{i}^{\dagger}\right)^{-1} \mathbf{Y}_{i}, \\
\mathbf{H}_{i+1} & =\mathbf{D}_{i} \mathbf{Y}_{i}^{-1} \mathbf{D}_{i}^{\dagger}\left(\mathbf{D}_{i+1}^{\dagger}\right)^{-1} \mathbf{Y}_{i+1} .
\end{aligned}
$$

To get the modal amplitudes we could recombine Eq. (33) and (24) to build a linear system $\mathbf{K A}=\mathbf{F}$, where all the unknowns, gathered in the vector $\mathbf{A}$, can be deduced from the right hand side of $\mathbf{F}$ arising from the incident wave. However, the inversion of such a system is, unfortunately, subject to round-off errors due to the presence of strongly evanescent waves. Because of these limitations, an alternative iterative procedure, proposed by Cummings ${ }^{25}$ and used successfully by the authors for silencer modeling ${ }^{21}$, is adopted here.

The idea is to treat each interface separately by considering all incoming waves as known quantities. At the first iteration, the unknown incoming waves $\left[\mathbf{B}^{i}, \mathbf{A}^{i+1}\right]^{\mathrm{t}}$ are fixed at zero or initialized by a previous frequency step solution. The systems given in Eq. (33) and/or in (24) are then solved for the outgoing waves $\left[\mathbf{A}^{i}, \mathbf{B}^{i+1}\right]^{t}$. This produces an initial value for the incoming waves on the next interface and so on. This loop over the interface stops when the relative error on modal amplitudes is smaller than $10^{-6}$ between two consecutive iterations. In practice, around $10 \times N_{\mathscr{L}}$ iterations are required. The factorization of the diagonal matrix $\mathbf{E}_{X}$, containing all propagation factors, is one of the main advantages of this iterative process. Indeed, its condition number is very large due to the combination of $x_{2}>0$ and $x_{2}<0$ traveling 'evanescent' waves. Thus, inverting $\mathbf{E}_{X}$ separately minimizes the effects of possible round-off errors. Furthermore, the scattering matrices $\mathbf{X}_{i}\left(i=0, \ldots, N_{\mathscr{L}}\right)$ are well-conditioned and the condition number can be enhanced by a careful choice of the weighting function in order to maximize their diagonal terms ${ }^{26}$ as already discussed in section II.C.1.

Once the wave amplitudes have been evaluated, the integration of the acoustic intensity leading to the energy balance can be applied. The integration is carried out over the periodic cell using the orthogonality relation of the Floquet modes given in (4). The energy reflection coefficient $^{18} \mathcal{R}$ is defined as the ratio of the scattered power in the $x_{2}$ direction

$$
\mathcal{P}_{r}=\sum_{n \in \mathbb{Z}} \mathfrak{R e}\left\{\beta_{n}\right\}\left|A_{n}^{0}\right|^{2} /\left(\rho^{0} \omega\right),
$$

to the incident power

$$
\mathcal{P}_{i}=\left|A_{0}\right|^{2} k_{2} d /\left(\rho^{0} \omega\right) .
$$

In practice, the number of propagating modes in $\Omega^{0}$ is very small and it is often reduced to the fundamental mode $n=0$ and the first mode $n= \pm 1$.
TABLE I. Material properties used in numerical tests. With the porosity $\phi$, flow resistivity $\sigma$, the tortuosity $\alpha_{\text {inf }}$, the viscous and thermal characteristic lengths $\Lambda$ and $\Lambda^{\prime}$.

\begin{tabular}{ccccccc}
\hline \hline Material & $\phi$ & $\begin{array}{c}\sigma \\
{\left[\mathrm{Nm}^{-4} \mathrm{~s}\right]}\end{array}$ & $\begin{array}{c}\alpha_{\text {inf }} \\
-\end{array}$ & $\begin{array}{c}\Lambda \\
{[\mu \mathrm{m}]}\end{array}$ & $\begin{array}{c}\Lambda^{\prime} \\
{[\mu \mathrm{m}]}\end{array}$ & Ref. \\
\hline Beads & 0.4 & 11,204 & 1.37 & 148 & 444 & {$[27,28]$} \\
Fireflex & 0.95 & 8,900 & 1.42 & 180 & 360 & {$[10]$} \\
RGW2 & 0.94 & 135,000 & 2.1 & 49 & 166 & {$[3]$} \\
Metal foam & 0.99 & 6,916 & 1.17 & 100 & 245 & - \\
\hline \hline
\end{tabular}

Thanks to the conservation of the energy, the absorbed power is given by $\mathcal{P}_{a b s}=\mathcal{P}_{i}-\mathcal{P}_{r}$. This yields the absorption coefficient

$$
\alpha=\frac{\mathcal{P}_{a b s}}{\mathcal{P}_{i}}=1-\mathcal{R} .
$$

\section{VALIDATION AND ACCURACY}

In this section the validation and the accuracy of the proposed method is investigated. Firstly, the computational efficiency is studied for the case of a porous layer with rectangular and circular inclusions. Secondly, an experimental validation is performed to check ability of the method to describe accurately the acoustic behavior of a finite size porous layer with inclusions composed of a number of periodic cells.

\section{A. Convergence study on rectangular shaped inclusions}

In this example, we consider porous and rigid rectangular inclusions embedded in metal foam (see Table. I). Because no solution is available in the literature for this geometry, the reference solutions are computed using a finite element model. These computations are carried out using Lagrange quadratic finite elements in the fluid and porous domains as described in Ref. 12. The nonreflecting boundary conditions are implemented using the Dirichlet to Newman (DtN) approach based on Eq. (4) to avoid problems when the wavelength is high compared with the computational domain size. The reference absorption computations $\hat{\alpha}$ are performed with a mesh of around 280,000 nodes. It can be noted that an equivalent meshing size leads to 8 digits accuracy in the predicted absorption coefficient for homogeneous porous (i.e. without inclusion). In this study the inclusion height was fixed to $15 \mathrm{~mm}$, and two widths, $a=15$ and $a=5 \mathrm{~mm}$, were tested. In both cases, the inclusions were located in the center of the $20 \times 20 \mathrm{~mm}$ periodic cell. These configurations have been tested at $5000 \mathrm{~Hz}$ for 2 incident wave angles, $\theta=0$ and $\theta=\pi / 3 \mathrm{rad}$.

With a porous inclusion (RGW2 wool, see Table. I), the present method has shown an exponential convergence, and a relative error $\mathcal{E}=\frac{|\alpha-\hat{\alpha}|}{\hat{\alpha}}$ below $1 \%$ has been achieved in all tested configurations with 3 modes and the classical matching scheme. With a perfectly rigid inclusion, the classical matching scheme is not numerically 

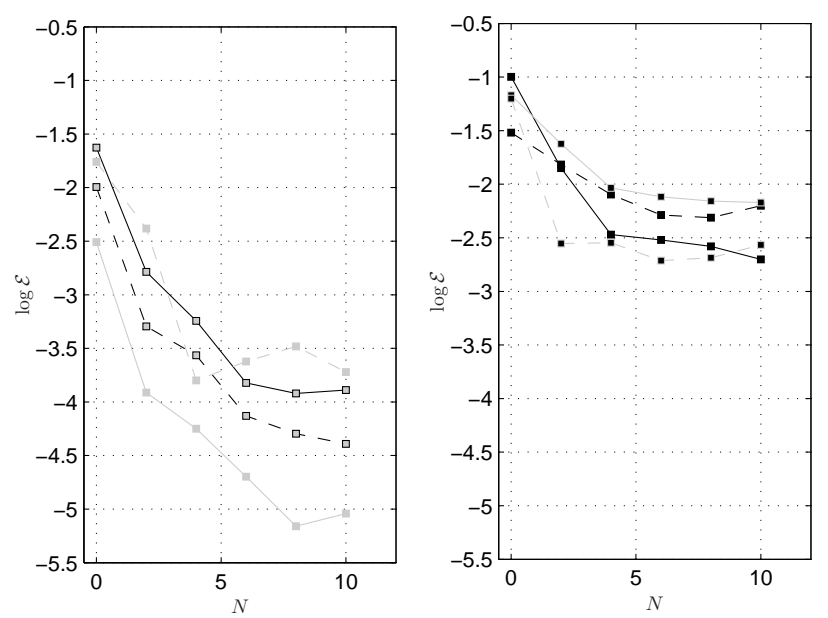

FIG. 3. Convergence of the proposed method for a small $(a / d=0.25,--)$ and larger $(a / d=0.75,-)$ inclusion size. The black lines stand for the normal incidence and the gray ones for $\theta=\pi / 3$. The black m-markers stand for the rigid inclusion (see on the right) and the gray $\square$-markers for the RGW2 porous inclusion (see on the left).

stable for large rigid inclusions, and it was not possible to get a relative error below $5 \%$ without accounting for at least thirty modes in the summation. It is known that the number of modes could be adapted according to the section ratio ${ }^{13}$ to enhance the convergence. However, the re-expansion scheme, described in sec II.C.2, was adopted because it seems faster and more robust. With this scheme, an error smaller than $1 \%$ can be achieved with only 3 modes. This accuracy seems sufficient for the applications proposed in this paper.

\section{B. Validation with the multipole method}

A comparison with the results presented in Ref. 10 (see Fig. 3 and 4 inside) for rigid circular inclusions has been performed. This kind of geometry can be tackled with the proposed method despite the fact that it is not the more appropriated technique. The multipole ${ }^{10}$ method or the method of fundamental solutions ${ }^{29}$ constitute better approaches for circular shape inclusion. Here, the circular inclusion of radius $a$ is discretized by 11 layers of rigid rectangular inclusions (see Fig. 4), and $N+1=7$ modes are taken into account in each layer. This level of discretization enables to achieve a good agreement in the frequency range up to $13 \mathrm{kHz}$ with the results of Groby et al. ${ }^{10}$ obtained for a similar problem as illustrated on Fig. 5. The presence of such inclusions can enhance the absorption of the Fireflex foam (see Table I), particularly around $2674 \mathrm{~Hz}$ where an absorption peak is obtained below the $1 / 4$-wavelength resonance frequency, $f_{\lambda / 4}$. This behavior can be explained by the excitation of a complex trapped mode ${ }^{10}$. At this frequency, the sound pressure maximum is localized near the rigid backing as depicted in Fig. 4. With the proposed modal approach, this rigid scatterer can be easily replaced by glass wool circular in-

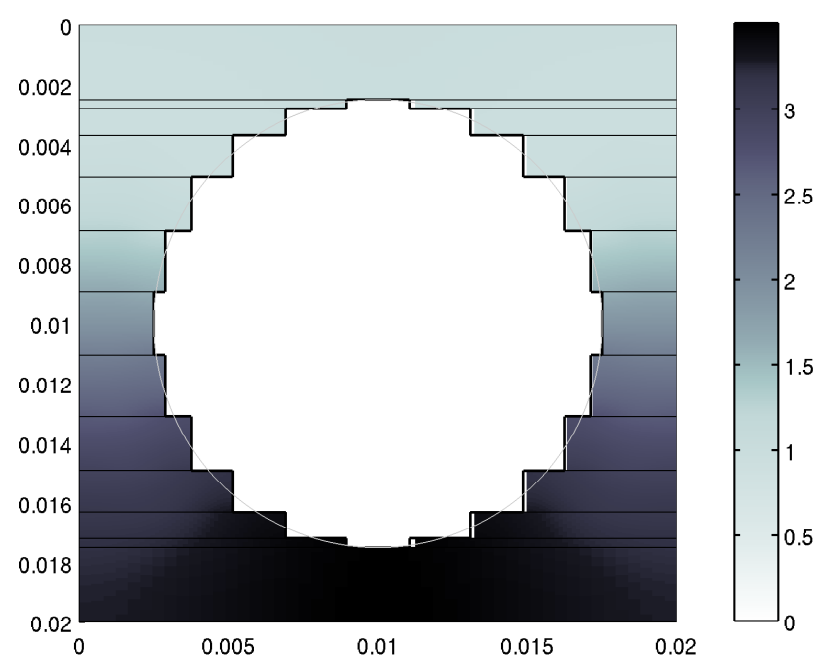

FIG. 4. Pressure modulus with the layered rigid circular inclusions at $2674 \mathrm{~Hz}$ (first absorption peak of Fig. 5). The horizontal lines stand for the layer interfaces.

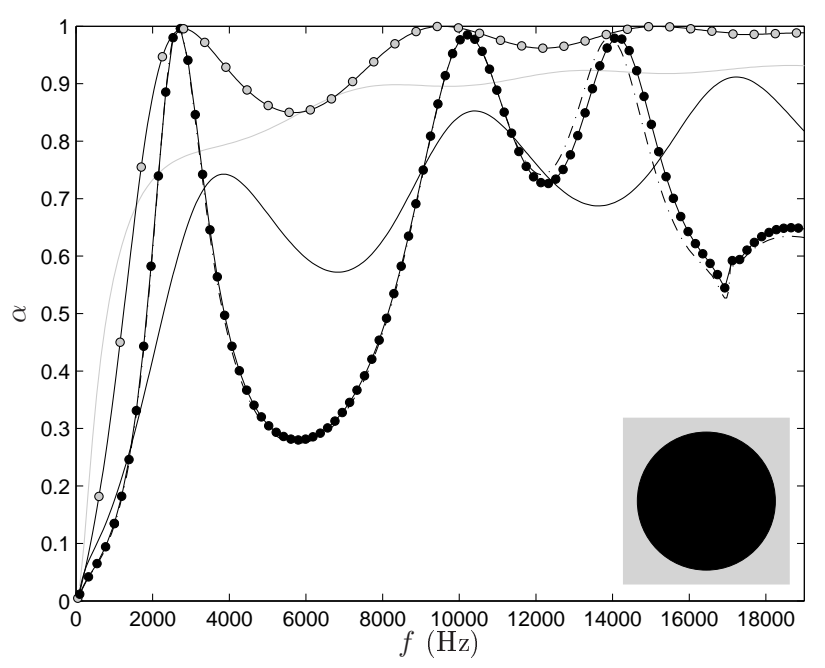

FIG. 5. Comparison and illustration of the proposed method with circular inclusions $(a=1.5 \mathrm{~cm})$ on a $2 \times 2 \mathrm{~cm}$ cell with 11 Layers. - homogeneous Fireflex material, - homogeneous RGW2 material, • circular rigid inclusion, -- - circular rigid inclusion from Ref. 10, - circular RGW2 inclusion.

clusions of the same radius (RGW2 wool, see Tab. I). Obviously, such results can also be obtained with slight modifications to the multipole method ${ }^{10}$. Above 1500 $\mathrm{Hz}$, this new heterogeneous material exhibited better absorbing properties than any of its constituent materials. It appears that the absorption properties of a heterogeneous porous material are only slightly sensitive to the inclusion shape, e.g. the presence of square inclusions with the same area leads to a similar absorption curve.

\section{Experimental validation}

An experimental validation has been performed in the anechoic chamber available in LAUM (Laboratoire 
d'Acoustique de l'Université du Maine, France). The experimental setup is illustrated in Figs. 6 and 7 . The tested sample was formed with 25 identical elementary cells of $20 \mathrm{~mm}$ width and of $21.5 \mathrm{~mm}$ height. These cells were created with a periodic array of $500 \mathrm{~mm}$ long, square aluminum bars which were backed by a $4 \mathrm{~mm}$ thick aluminum plate attached to a $20 \mathrm{~mm}$ thick wooden base. Fig. 6 illustrates schematically the arrangement of these bars and the periodic grating which this arrangement recreated. The experimental setup shown in this figure had the following dimensions: $a=15 \mathrm{~mm}$, $h=21.5 \mathrm{~mm}, t_{1}=2.5 \mathrm{~mm}, t_{2}=4 \mathrm{~mm}$ and $d=20 \mathrm{~mm}$. The simulated periodic grating $(500 \times 500 \mathrm{~mm})$ was filled with $2 \mathrm{~mm}$ glass beads (see Fig. 7) to the required thickness. Additional beads were spread around the sample to cover the whole wooden plate $(1 \times 1 \mathrm{~m})$. The use of glass beads enabled to create a porous filling around various inclusion shapes. This medium had the basic acoustical and non-acoustical characteristics which were relatively easy to predict. The Johnson-Champoux-Allard properties for these beads are summarized in Tab. I. The values of these parameters were borrowed from Refs. 27, 28.

A parametric source array ${ }^{30}$ (using parametric demodulated ultrasonic waves) was used to create a highly directional plane wave normal to the grating at audible frequencies to control the diffraction from the edges of the periodic grating. The distance between the center of the grating and parametric source array was $1.3 \mathrm{~m}$. A dynamic signal analyzer (SR785) was used to drive the parametric source array with a swept sine. A pair of phase-matched microphones (1/4" microphones B\&K 4938 with B\&K 2670 preamplifiers and Nexus 4-channel amplifier) was installed at the center of the grating. The microphone elevations above the center of grating were $h_{1}=8 \mathrm{~mm}$ and $h_{2}=28 \mathrm{~mm}$, respectively. Below the first cut-off frequency of the Floquet mode (around 17,000 Hz for the $2 \mathrm{~cm}$ cell), the energy reflection coefficient was deduced from

$$
\mathcal{R}(\omega)=\left|\mathrm{e}^{-2 \mathrm{i} k^{0} h_{1}} \frac{\mathrm{e}^{-\mathrm{i} k^{0}\left(h_{2}-h_{1}\right)}-H(\omega)}{H(\omega)-\mathrm{e}^{\mathrm{i} k^{0}\left(h_{2}-h_{1}\right)}}\right|^{2},
$$

and the absorption coefficient was computed with Eq. (38). Here $H(\omega)=\frac{p^{0}\left(0, h_{2}\right)}{p^{0}\left(0, h_{1}\right)}$ denotes the frequency transfer function between the two microphones located in the middle of the material sample. An analog $20 \mathrm{kHz}$ lowpass Butterworth/Bessel multichannel filter from KrohnHite Corporation model \# 3945 was used to remove the residual ultrasonic waves emitted by the parametric array.

The comparison of the simulations and experimental data is presented in Fig. 8 for the homogeneous beads and for an array of rigid, square inclusions. In all cases the general behavior of the absorption curves is closely predicted. A good agreement is obtained for the frequency of the first absorption peak and for its amplitude. The discrepancies with the square inclusions are in line with the results obtained in the case of the homogeneous porous layer. The presence of oscillations in the acoustic absorption spectrum is clearly visible in the case of the square inclusions. This can be explained by the edge diffraction effect which relates to the finite size of the simulated periodic grating. This effect is not taken into account by Eq. (39).

In the homogeneous case, the $1 / 4$-wavelength resonance accounts for the absorption peak around $f_{\lambda / 4}=$ $3 \mathrm{kHz}$. The use of square rigid inclusions allows for the measurement of a high absorption peak at $2041 \mathrm{~Hz}$, which is below the predicted value of $f_{\lambda / 4}$. At this frequency, the absolute part of the sound pressure computed with the proposed method is high and it is localized near the backing wall. In this case, the absorption coefficient at the higher frequencies is relatively poor. An estimation of the absorption peak frequency $f_{\alpha}$ accurate within a few percent can be obtained with a modified expression for the frequency of the $1 / 4$-wavelength resonance

$$
f_{\alpha} \approx \frac{c}{4 h^{\prime}} \approx 2200 \mathrm{~Hz}
$$

where $c$ is the sound speed in the beads and $h^{\prime}=$ $\left(h-t_{2} / 2\right)+a / 2+t_{1} / 2=28.25 \mathrm{~mm}$ corresponds to the apparent length along the square inclusion (see Fig. 6).

\section{NUMERICAL RESULTS ON SQUARE SCATTERER}

Numerical calculations of the absorption coefficient have been performed for various inclusion types and shapes. The effect of the angle incidence on the absorption coefficient of this system has also been studied. All computations were performed for metal foam which properties are presented in (see Tab. I).

\section{A. Inclusion types}

In this section, we are interested in square inclusions located in the center of the periodic cell $(20 \times 20 \mathrm{~mm})$ and excited by a plane wave propagating at the normal angle of incidence. The inclusion height $a$ was varied from 1 $\mathrm{mm}$ to $18 \mathrm{~mm}$ in a $1 \mathrm{~mm}$ step. It was assumed that the inclusions were square, rigid and/or filled with air or with another porous material with contrasting properties (e.g. RGW2). The predicted absorption coefficient for these configurations is shown in Fig. 9.

The results suggest that the air-filled inclusions embedded in this metal foam do not enhanced the acoustic absorbing behavior and that bigger air-filled inclusions result generally in a lower absorption coefficient. This result is predictable, because it is known ${ }^{3}$ that air gaps are efficient only for highly resistive and weakly tortuous porous material such as RGW2. The RGW2-filled inclusions case is more effective. If the inclusions are small, then the behavior is dominated by the metal foam matrix. On the contrary, when the inclusions are large, then the behavior is close to that expected for an homogeneous layer of RGW2 wool. Between these two extreme configurations, the absorption of the heterogeneous material is generally better than the absorption of each of the two homogeneous materials considered separately. This stands for the range of the square inclusion height considered in this study $(a \in[10,18] \mathrm{mm})$. 


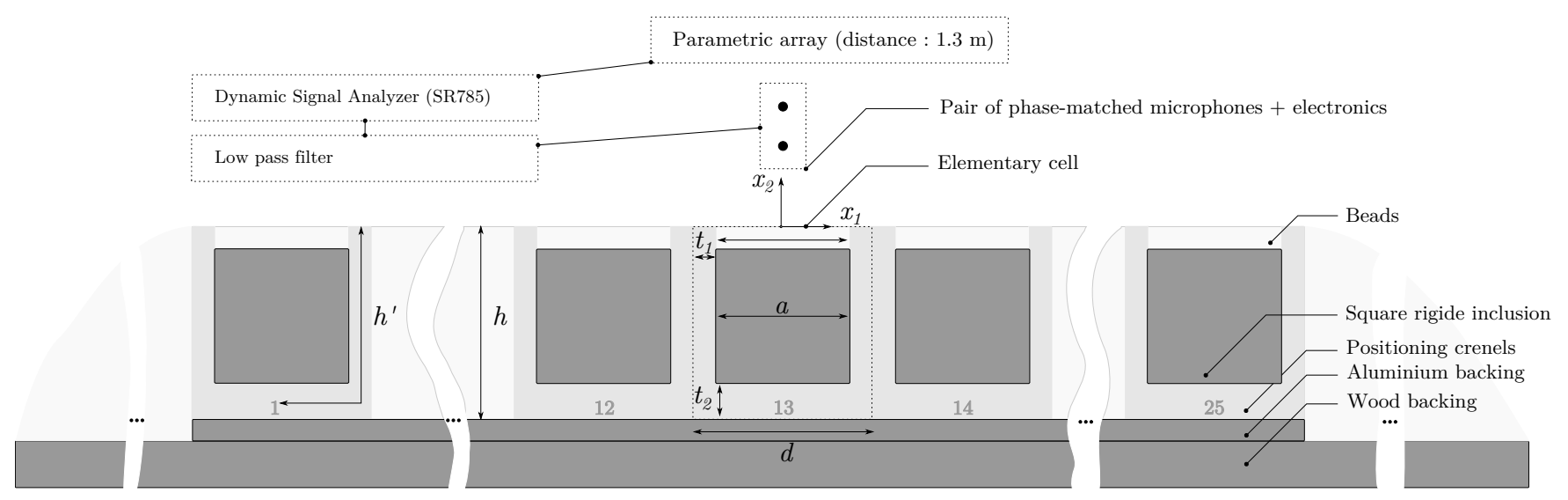

FIG. 6. Schematic description of the experimental setup with the square section.

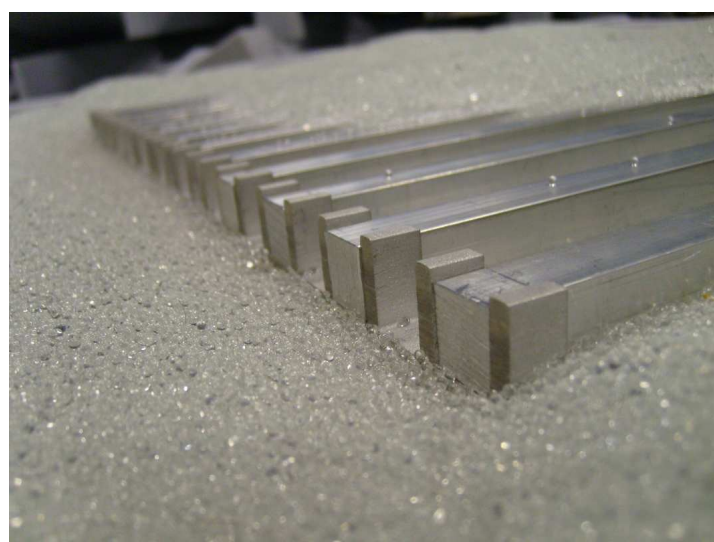

FIG. 7. Picture of the experimental setup before total beads and inclusions filling.

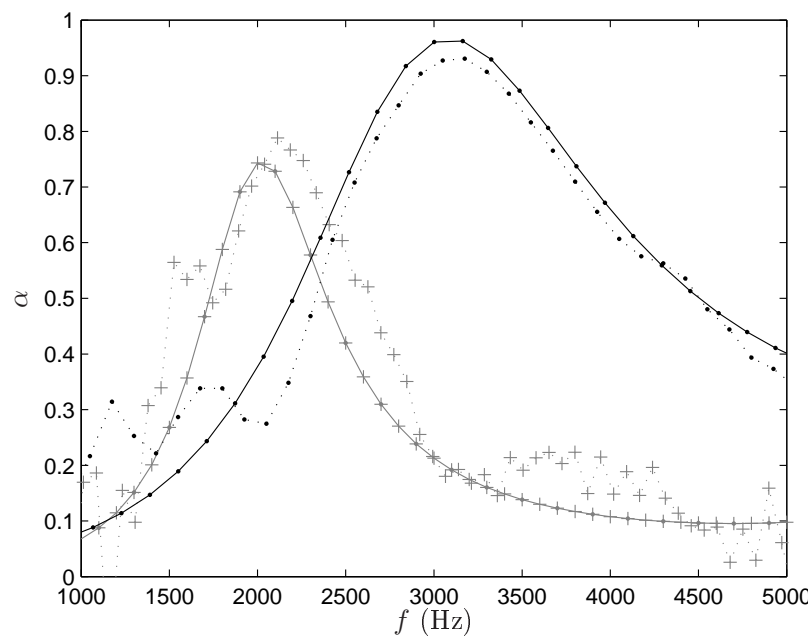

FIG. 8. Comparison between simulations (solid line) and measurements (dotted line) for square shape inclusion $(+)$ and homogeneous beads $(\cdot)$.
The results from this modeling work also show that the absorption coefficient is not too sensitive to the geometry of the embedded porous shape and that the filling ratio seems to be the most significant parameter which accounts for any improvement. These results are in line with those obtained by the homogeneization method $^{8}$ or by Nennig et al. ${ }^{29}$ for poroelastic scatterers embedded in air.

For the rigid inclusions case, the results are similar to those presented with the circular inclusions in section III.B. If the inclusion's size is sufficiently large, a peak in the absorption spectrum emerges. Hence, in the case of the considered layer configuration, the absorption coefficient may be enhanced between 2000 and $4000 \mathrm{~Hz}$. The absorption coefficient reaches a minimum around 5 $\mathrm{kHz}$. Groby et al. ${ }^{10}$ have explained this behavior by the presence of a bandgap of the 'underlying' periodic array arising from the perfect reflection on the hard backing. Within this bandgap most of the acoustic energy is reflected backward, i.e. back in the domain $\Omega^{0}$, leading to a poor absorption.

\section{B. The effect of the inclusion position}

As already shown by Groby et al. ${ }^{10}$, that the inclusion position in the periodic cell can be a sensitive parameter. For a given square inclusion $(a=15 \mathrm{~mm})$, the influence of the inclusion height, $t_{2}$, and the periodic cell width, $d$, are illustrated in Fig. 10. It can be shown that the absorption frequency increases for larger values of the cell period, $d$. Moreover, the peak amplitude reaches a maximum for $d \approx 30 \mathrm{~mm}$. Note that sudden variation of the absorption coefficient (the so called Wood's anomalies) can be observed at the first cut-off frequency of the Floquet mode ${ }^{18}$ when $d$ is sufficiently large.

The influence of the inclusions height, $t_{2}$, is essentially related to the absorption peak frequency and has nearly no influence on the peak amplitude. When the height $t_{2}$ increases, the absorption peak frequency decreases. Once again, this can be explained ${ }^{10}$ by the presence of the 'underlying' periodic array formed by the inclusions images 


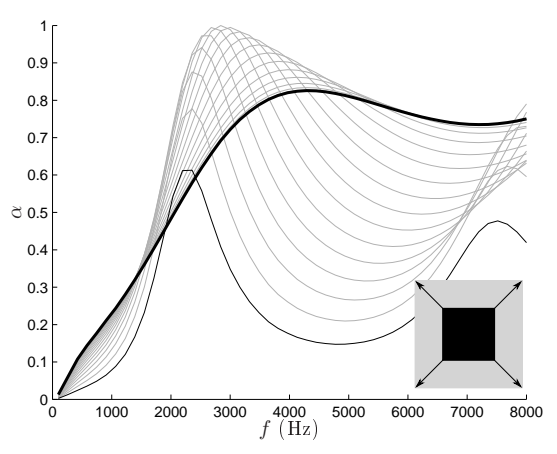

(a) rigid

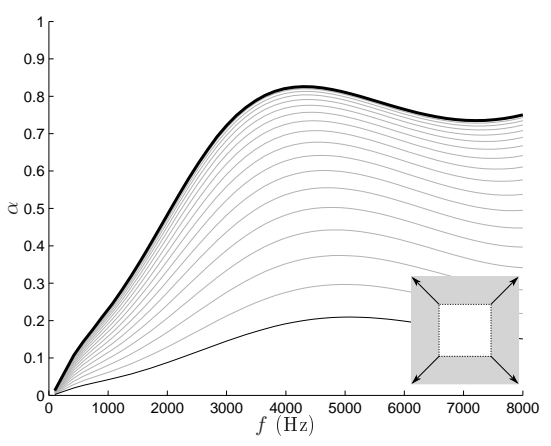

(b) air

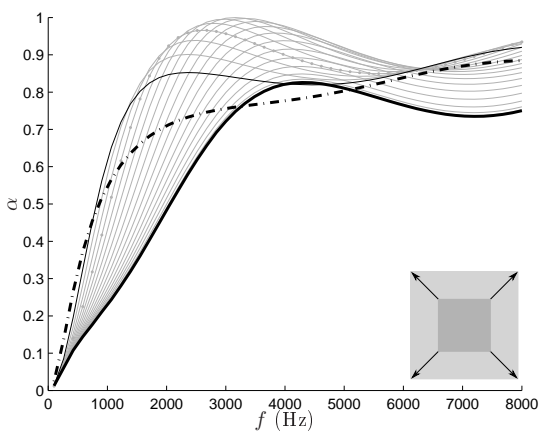

(c) porous

FIG. 9. Effect of the size $a$ of a square inclusion on the absorption coefficient. The inclusion size ranges from 1 to $18 \mathrm{~mm}$ in $1 \mathrm{~mm}$ step. (- ) stands for the upper bound $(18 \mathrm{~mm})$ of the variation interval. The reference homogeneous metal foam absorption is denoted by ( $(\boldsymbol{)})$ and the RGW2 wool by (- $-\boldsymbol{~ )}$. Rigid inclusion (a), Air inclusion (b), and RGW2 porous inclusion (c).

with respect to the hard backing. When $t_{2}$ increases, the period of the image sonic crystal increases, pushing peaks towards the low frequencies.

\section{Effect of the angle of incidence}

The Floquet-Bloch formalism presented in this work enables to study the effect of the angle of incidence $\theta$. The low frequency absorption coefficient of a homogeneous porous layer increases with $\theta$. However, the behavior in the medium and high frequencies depends strongly on the material properties. For example, in the case of metal foam the absorption coefficient drastically decreases with $\theta$ above $2000 \mathrm{~Hz}$ in comparison with the normal incidence case (see Fig. 11). When the variation of the angle of incidence is small, then the absorption behavior is similar to that predicted for the normal incidence case. In contrast, when the angle of incidence is large (say $\theta>\pi / 3$ ), the absorption can change considerably.

With a rigid square inclusion, this general behavior remains unchanged but the effect of the high value of $\theta$ seems to be less significant and the absorption peak frequency typically increases with $\theta$.

\section{THE INFLUENCE OF THE INCLUSION SHAPE}

Squares or circles are not the optimal inclusions shapes because a large part of the porous matter is removed from the layer. As a result, its broadband absorption capabilities are partially lost. A better approach is to use 'open' or 'partially open' inclusions, such $\sqcup$ (as illustrated on Fig. 12), to increase the apparent tortuosity of the material and to add more trapped modes. A number of different configurations were studied in this work by changing the $\sqcup$-section orientation, e.g. $\sqcup, \sqcap$ or $\sqsubset$. The acoustic absorption coefficient for a heterogeneous porous layer with these types of inclusions was predicted with the proposed numerical method.

It was found that the absorption coefficient of a porous layer with rigid inclusions is more sensitive to the inclu-

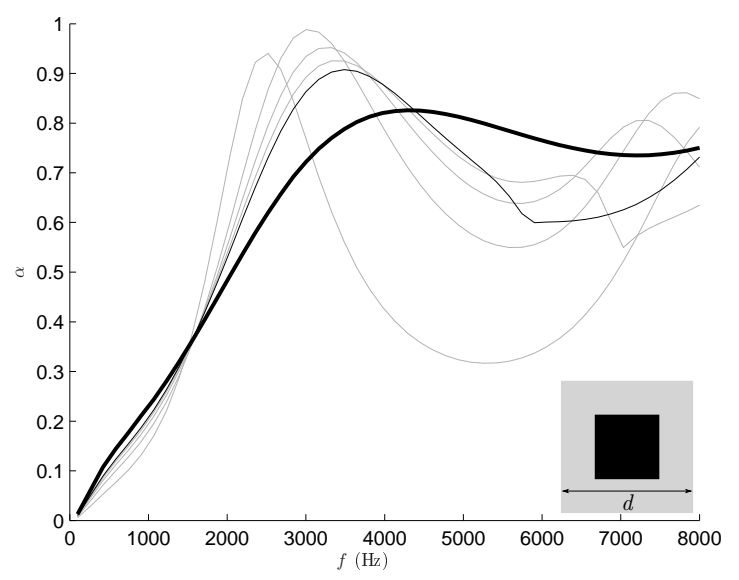

(a) $d$ from 20 by 10 to $60 \mathrm{~mm}$

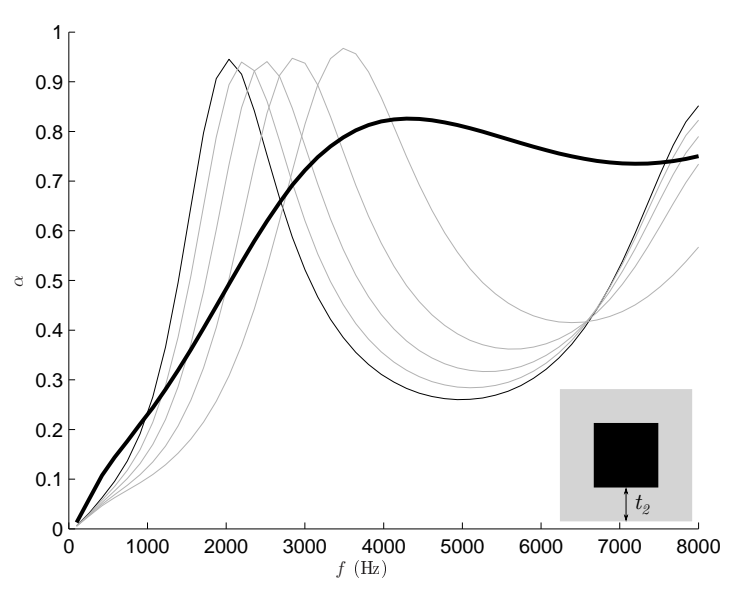

(b) $t_{2}$ from 0.5 by 1 to $4.5 \mathrm{~mm}$

FIG. 10. Influence of the elementary cell period $d$ (a) and of the inclusions height $t_{2}$ (b) on the absorption coefficient with rigid square inclusions (with $a=15 \mathrm{~mm}$ ) grating. The homogeneous metal foam absorption is denoted by ( $)$ in normal incidence. In all cases, (- ) stands for the upper bound of the variation interval. 


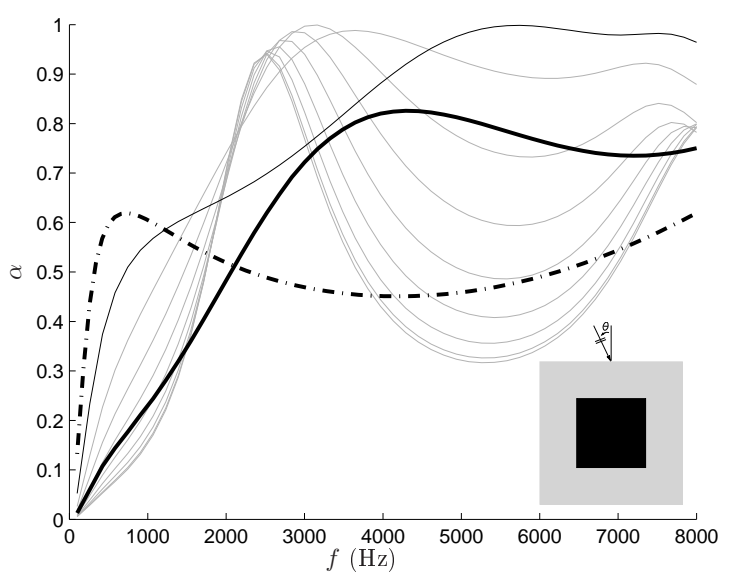

FIG. 11. Effect of the angle of incidence ( $\theta$ from 0 by 10 to $80^{\circ}$ ) on the absorption coefficient of a proous layer with rigid square inclusions (with $a=15 \mathrm{~mm}$ ). The homogeneous metal foam material is denoted by ( $(-)$ in normal incidence and by (-・ ) for $\theta=80^{\circ}$. The upper bound of the variation interval $\left(80^{\circ}\right)$ is denoted by $(-)$.

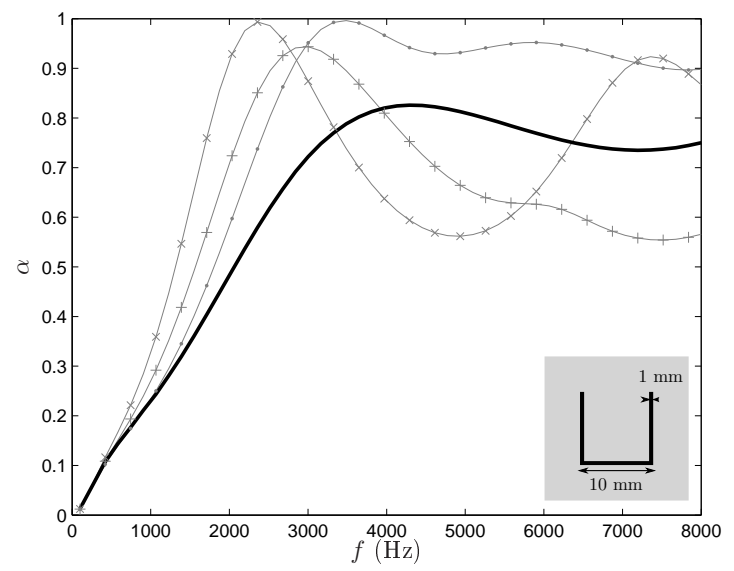

FIG. 12. Effect on the absorption coefficient of the orientation of a rigid $\sqcup$ inclusion embedded in the metal foam $(a=10 \mathrm{~mm}$, thickness $1 \mathrm{~mm})$. The $(\cdot)$ stand for the $\sqcup$, the $(x)$ for the $\sqcap$ and the $(+)$ for the $\sqsubset$ orientation. The homogeneous metal foam absorption is denoted by ( $(-)$.

sion shape than that in the case of the layer impregnated with fluid-filled or porous inclusions. For example, the presence of a $\sqcap$-section in a porous layer yields the lowest absorption peak frequency, i.e. nearly two times smaller than the $f_{\lambda / 4}$ frequency predicted for the uniform porous layer. At this frequency the sound pressure modulus near the wall of a rigid $\sqcap$-shape inclusion is relatively high and strongly localized. However, the absorption coefficient spectra oscillates significantly as a function of frequency.

With the upside-down configuration, i. e. $\sqcup$-shape, the absorbing coefficient can be greatly enhanced (by more than 20\%) across the entire frequency range. This is associated with the the presence of two absorption peaks caused by the existence of two trapped modes propagating in the porous layer. One of these modes relates to a localized pressure field between the inclusions and the backing wall. In the case of the second peak, the pressure modulus is localized near the wall inside the $\sqcup$ inclusion. The $\sqsubset$ orientation exhibits a behavior between the two previous cases.

\section{CONCLUSIONS}

In this article, a new mode matching scheme is proposed in order to study the acoustical behavior of a multilayer porous material in which periodic inclusions are embedded. The method is validated numerically and experimentally with rigid inclusions embedded in $2 \mathrm{~mm}$ beads. It has been shown that taking into account the inclusions geometry is necessary when the inclusions are rigid. The proposed method can tackle a large variety of shapes and inclusion types faster than a conventional FEM model.

This work is an attempt to investigate the influence of the shape of rigid scatterers embedded in a porous material. It has been shown that open shape inclusions (e.g. $\sqcup$-shape) are able to provide a better effect than inclusions of closed shapes, because less porous material is removed from the layer. These heterogeneous structures can exhibit better acoustic absorption behavior than homogeneous porous layers of identical thickness and provide an alternative to multi-layering.

Future work can focus on exploring new inclusion shapes or more simple shape combinations. In particular, rigid inclusions combined with metal foam is an interesting way to achieve a compact, efficient acoustic treatment for a harsh environment or when good structural behavior is required.

\section{Acknowledgment}

This work was in part financially supported by the FUI project REBECCA. The authors would like to thank their industrial partners Snecma and Snecma Propulsion Solide from Safran group.

The authors would like to thank Prof. Kirill V. Horoshenkov for his original idea about the beads for manufacturing the surrounding porous material and for his comments on this manuscript.

\section{APPENDIX A: RIGID FRAME MODEL}

Porous materials with a rigid skeleton (and quite regular pore shape), such as the porous material involved in this study, are well described by the Johnson-ChampouxAllard $^{1}$ (Chap. 5) equivalent fluid model. This equivalent fluid has the equivalent density $\left(e^{-i \omega t}\right)$.

$$
\rho=\frac{\alpha_{\infty} \rho^{0}}{\phi}\left[1+\mathrm{i} \frac{\sigma \phi}{\omega \rho^{0} \alpha_{\infty}} G_{J}(\omega)\right]
$$


and the bulk modulus,

$$
K=\frac{\gamma P^{0} / \phi}{\gamma-(\gamma-1)\left[1+\mathrm{i} \frac{8 \eta}{\Lambda^{\prime 2} \operatorname{Pr} \omega \rho^{0}}\left(1-\mathrm{i} \rho^{0} \frac{\omega \operatorname{Pr} \Lambda^{\prime 2}}{16 \eta}\right)^{1 / 2}\right]^{-1}} .
$$

Note that the domain indices $i, j$ are omitted for clarity. Here, $G_{J}(\omega)=\sqrt{1-\frac{4 \mathrm{i} \alpha_{\infty}^{2} \eta \rho^{0} \omega}{\sigma^{2} \Lambda^{2} \phi^{2}}}, \phi$ is the porosity, $\sigma$ is the flow resistivity, $\Lambda$ is the vicious length, $\Lambda^{\prime}$ is the thermal length, $\alpha_{\infty}$ is the tortuosity. Moreover, $\gamma$ is the air specific heat ratio and $P^{0}$ is the atmospheric pressure, $\operatorname{Pr}$ is the Prandtl number and $\eta$ is the dynamic viscosity. It is important to note, the viscosity is taken into account in the porous material pore but is neglected in the surrounding fluid.

1 J.-F. Allard, Propagation of Sound in Porous Media: Modeling Sound Absorbing Materials, 280pp (Chapman \& Hall, New York) (1993).

${ }^{2}$ N. Atalla, R. Panneton, F. C. Sgard, and X. Olny, "Acoustic absorption of macro-perforated porous materials", J. Sound Vib. 243, 659-678 (2001).

${ }^{3}$ F. C. Sgard, X. Olny, N. Atalla, and F. Castel, "On the use of perforations to improve the sound absorption of porous materials", Appl. Acoust. 66, 625-651 (2005).

${ }^{4}$ F. C. Sgard, F. Castel, and N. Atalla, "Use of a hybrid adaptive finite element/modal approach to assess the sound absorption of porous materials with mesoheterogeneities", Appl. Acoust. 72, 157-168 (2011).

5 J. S. Lee, Y. Y. Kim, J. S. Kim, and Y. J. Kang, "Twodimensional poroelastic acoustical foam shape design for absorption coefficient maximization by topology optimization method", J. Acoust. Soc. Am. 123, 2094-2106 (2008).

${ }^{6} \mathrm{X}$. Olny and C. Boutin, "Acoustic wave propagation in double porosity media", J. Acoust. Soc. Am. 114, 73-89 (2005).

7 F.-X. Bécot, L. Jaouen, and E. Gourdon, "Application of the dual porosity theory to irregularly shaped porous materials", Acta Acust. 94, 715-724 (2008).

${ }^{8}$ E. Gourdon and M. Seppi, "On the use of porous inclusions to improve the acoustical response of porous materials: Analytical model and experimental verification", Appl. Acoust. 71, $283-298$ (2010)

9 J.-P. Groby, A. Wirgin, L. De Ryck, and W. Lauriks, "Acoustic response of a rigid frame porous medium slab with a periodic set of inclusions", J. Acoust. Soc. Am. 126, 685-693 (2009).

10 J.-P. Groby, A. Duclos, O. Dazel, L. Boeckx, and W. Lauriks, "Enhancing absorption coefficient of a backed rigid frame porous layer by embedding circular periodic inclusions", J. Acoust. Soc. Am. 130, 3071-3780 (2011).

11 J.-P. Groby, A. Duclos, O. Dazel, L. Boeckx, and W. Lauriks, "Absorption of a rigid frame porous layer with periodic circular inclusions backed by a periodic grating", J. Acoust. Soc. Am. 129, 3035-3046 (2011).

12 J.-F. Allard, O. Dazel, G. Gautier, J.-P. Groby, and W. Lauriks, "Prediction of sound reflection by corrugated porous surfaces", J. Acoust. Soc. Am. 129, 1696-1706 (2011).

13 J. Kergomard and A. Garcia, "Simple discontinuities in acoustic waveguides at low frequencies: Critical analysis and formulae", J. sound Vib. 114, 465-479 (1987).

14 A. Abramowitz and I. Stegum, Handbook of Mathematical functions, 1043pp, (Dover, New York) (1965).
15 D. Gottlieb, C.-W. Shu, A. Solomonoff, and H. Vanderen, "On the Gibbs phenomenon I: Recovering exponential accuracy from the Fourier partial sum of a nonperiodic analytic function", J. Comput. Appl. Math. 43, 81-98 (1992).

16 V. Evans and M. Fernyhough, "Edge waves along periodic coastlines. Part 2", J. Fluid Mech. 297, 307-325 (1995).

17 D. Homentcovschi and R. N. Miles, "A re-expansion method for determining the acoustical impedance and the scattering matrix for the waveguide discontinuity problem", J. Acoust. Soc. Am. 128, 628-638 (2010).

18 C. M. Linton and I. Thompson, "Resonant effects in scattering by periodic arrays", Wave motion 44, 167-175 (2007).

19 C. M. Linton and P. McIver, Handbook of Mathematical Techniques for Wave Structure Interactions, 320pp (Chapman \& Hall/CRC, Boca Raton) (2001).

20 J.-P. Groby, W. Lauriks, and V. T. E., "Total absorption peak by use of a rigid frame porous layer backed by a rigid multi-irregularities grating", J. Acoust. Soc. Am. 127, 2865-2874 (2010).

${ }^{21}$ B. Nennig, E. Perrey-Debain, and M. Ben Tahar, "A mode matching method for modelling dissipative silencers lined with poroelastic materials and containing mean flow", J. Acoust. Soc. Am. 128, 3308-3320 (2010).

22 L. C. Botten, M. S. Craig, R. C. McPhedran, J. L. Adams, and R. J. Andrewartha, "The finitely conducting lamellar diffraction gratting", Opt. Acta 28, 1087-1102 (1981).

23 J. B. Lawrie and R. Kirby, "Mode-matching without rootfinding: Application to a dissipative silencer", J. Acoust. Soc. Am. 119, 2050-2061 (2006).

24 S. Elhay and J. Kautsky, "Algorithm 655: IQPACK: FORTRAN subroutines for the weights of interpolatory quadratures", ACM Trans. Math. Softw. 13, 399-415 (1987).

25 A. Cummings and I.-J. Chang, "Sound attenuation of a finite length dissipative flow duct silencer with internal mean flow in the absorbent", J. Sound Vib. 127, 1-17 (1987).

26 G. Gabard and R. J. Astley, "A computational modematching approach for sound propagation in threedimensional ducts with flow", J. Sound Vib. 315, 11031124 (2008).

27 O. Dazel and V. Tournat, "Nonlinear Biot waves in porous media with application to unconsolidated granular media", J. Acoust. Soc. Am. 127, 692-702 (2009).

28 J.-F. Allard, M. Henry, and J. Tiziantel, "Sound propagation in air-saturated random packings of beads", J. Acoust. Soc. Am. 104, 2004-2007 (1998).

29 B. Nennig, E. Perrey-Debain, and J.-D. Chazot, "The method of fundamental solutions for acoustic wave scattering by a single and a periodic array of poroelastic scatterers", Eng. Anal. Bound. Elem. 35, 1019-1028 (2010).

30 B. Castagnède, M. Saeid, A. Moussatov, V. Gusev, and V. Tournat, "Reflexion and transmission at normal incidence onto air-saturated porous materials and direct measurements based on parametric demodulated ultrasonic waves", Ultrasonics 44, 221-229 (2006). 